\title{
Nonlinear Phase-Phase Cross-Frequency Coupling Mediates Communication between Distant Sites in Human Neocortex
}

\author{
Felix Darvas, ${ }^{1}$ Kai J. Miller, ${ }^{3}$ Rajesh P. N. Rao, ${ }^{2}$ and Jeffrey G. Ojemann ${ }^{1}$ \\ ${ }^{1}$ Department of Neurological Surgery and ${ }^{2}$ Paul G. Allen Center for Computer Science and Engineering, University of Washington, Seattle, Washington \\ 98195, and ${ }^{3}$ Department of Physics, Neural Systems Laboratory, Harborview Hospital, University of Washington, Seattle, Washington 98104
}

\begin{abstract}
Human cognition is thought to be mediated by large-scale interactions between distant sites in the neocortex. Synchronization between different cortical areas has been suggested as one possible mechanism for corticocortical interaction. Here, we report robust, directional cross-frequency synchronization between distant sensorimotor sites in human neocortex during a movement task. In four subjects, electrocorticographic recordings from the cortical surface revealed a low-frequency rhythm $(10-13 \mathrm{~Hz})$ that combined with a higher frequency $(77-82 \mathrm{~Hz})$ in a ventral region of the premotor cortex to produce a third rhythm at the sum of these two frequencies in a distant motor site. Such cross-frequency coupling implies a nonlinear interaction between these cortical sites. These findings demonstrate that task-specific, phase-phase coupling can support communication between distant areas of the human neocortex.
\end{abstract}

Key words: synchronization; motor cortex; EEG; frequency; directional; human

\section{Introduction}

Synchronization of cortical areas in the human brain plays an important role in the understanding of the computational basis for human cognition (Kahana, 2006). Phase synchronization at single frequencies between distant areas is thought to be relevant to how these regions interact and such synchronization (e.g., in the motor system) has been well studied (Ohara et al., 2001; Gross et al., 2002; Simoes et al., 2003). However, coupling across frequencies would allow for much more complex corticocortical interactions (Buzsáki and Draguhn, 2004) and has been examined in brain signals (Shils et al., 1996; Schack et al., 2002; Palva et al., 2005; Canolty et al., 2006). Cross-frequency coupling in the human brain suggests nonlinear interaction, and it is speculated that such interactions play a crucial role in the coordination of complex cortical computation. A possible role for crossfrequency coupling between distant sites could be information exchange as a result of distributed cortical processing of complex behavior. Here, we demonstrate time-dependent crossfrequency phase coupling between distant sites in the motor area during execution of a simple finger movement task, using a biphase-locking synchronization measure. This measure is sensitive to quadratic (i.e., multiplicative) interactions between signals. In contrast to harmonic m:n phase coupling of single frequency bands, which can also be detected by biphase locking, this measure can detect coupling between any two frequencies. Unlike bicoherence, which has been previously used in cross-

\footnotetext{
Received Aug. 4, 2008; revised Sept. 15, 2008; accepted Nov. 26, 2008.

This work was supported by National Institutes of Health Grants EB007362 and T32-NS07144 and National Science Foundation Grants 0642848 and 0622252.

Correspondence should be addressed to Dr. Felix Darvas, Department of Neurological Surgery, University of Washington, Paul G. Allen Center for Computer Science and Engineering, Box 352350, Seattle, WA 98195. E-mail: fdarvas@u.washington.edu.

DOI:10.1523/JNEUROSCI.3688-08.2009

Copyright $\odot 2009$ Society for Neuroscience $\quad 0270-6474 / 09 / 290426-10 \$ 15.00 / 0$
}

frequency analysis (Schack et al., 2002), the biphase-locking measure is a pure phase synchronization measure (i.e., it is not affected by amplitude covariation). Multiplication of neural signals, as measured by biphase locking, can be seen as a fundamental aspect of higher cortical function and has been shown to exist on a single-neuron level (Gabbiani et al., 2002). Other roles for multiplicative interaction can be found in neuronal control circuits, such as phase-locked loops (Ahissar and Kleinfeld, 2003; Zacksenhouse and Ahissar, 2006) for sensorimotor control, but it could also serve as a natural mechanism for large-scale phase modulation of neuronal ensembles. These modulations exist in ongoing cortical activity and increase specifically during cognitive tasks.

Previous studies have analyzed phase-amplitude relationships or amplitude-amplitude relationships, but recent studies have shown that phase-phase relationships across cortical regions are of equal importance (Rudrauf et al., 2006). Although amplitude changes can take place because of an increase in local synchrony, they can also change with increased overall neuronal activity and thus do not, by necessity, reflect synchronization. By the same token, phase synchronization between distant neuronal populations can take place without local amplitude changes (Palva and Palva, 2007). Moreover, phase-phase relationships can be seen as correlates of spike timing, in which synchronization of the phases directly reflects neuronal communication (Fries, 2005).

Previous human electrocorticographic (ECoG) studies (Mormann et al., 2005; Canolty et al., 2006) have shown that there exists modulation of high frequencies in the gamma band $(>40$ $\mathrm{Hz}$ ) by lower frequencies during behavioral tasks. Specifically, it has been shown that low $(8-32 \mathrm{~Hz})$ and high $(76-100 \mathrm{~Hz})$ frequencies play complementary roles during finger movement (Miller et al., 2007a). The high-frequency increases appear to reflect more local behavior and are distinct from the low- 
Table 1. Subject demographics

\begin{tabular}{llllll}
\hline Subject & Side & Age (years) & Handedness & Gender & Location of grid \\
\hline 1 & Right & 21 & Right & S & Frontal \\
2 & Left & 18 & Right & Medial frontal & Frontal \\
3 & Left & 32 & Right & A & Frontal temporal \\
4 & Left & 46 & Right & F & Frontal \\
\hline
\end{tabular}
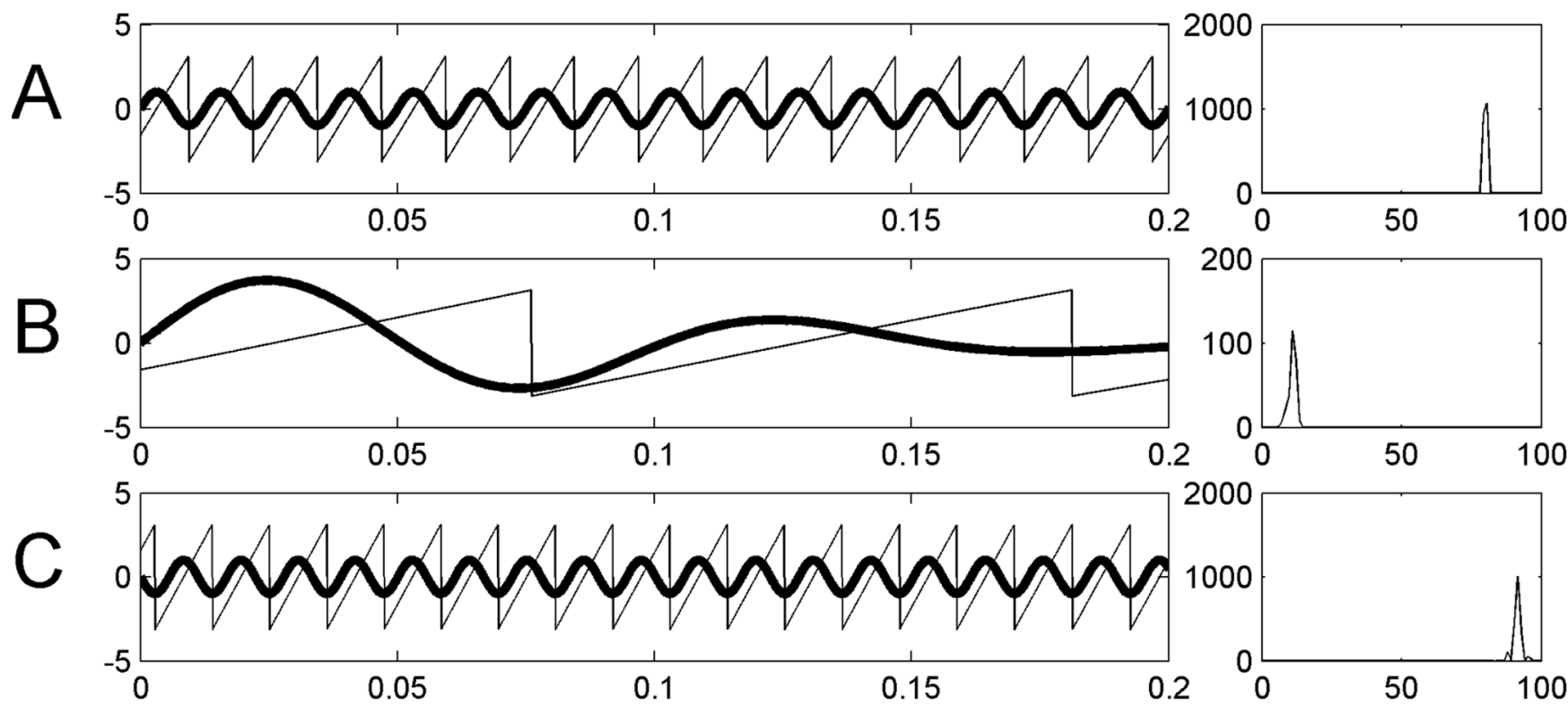

D
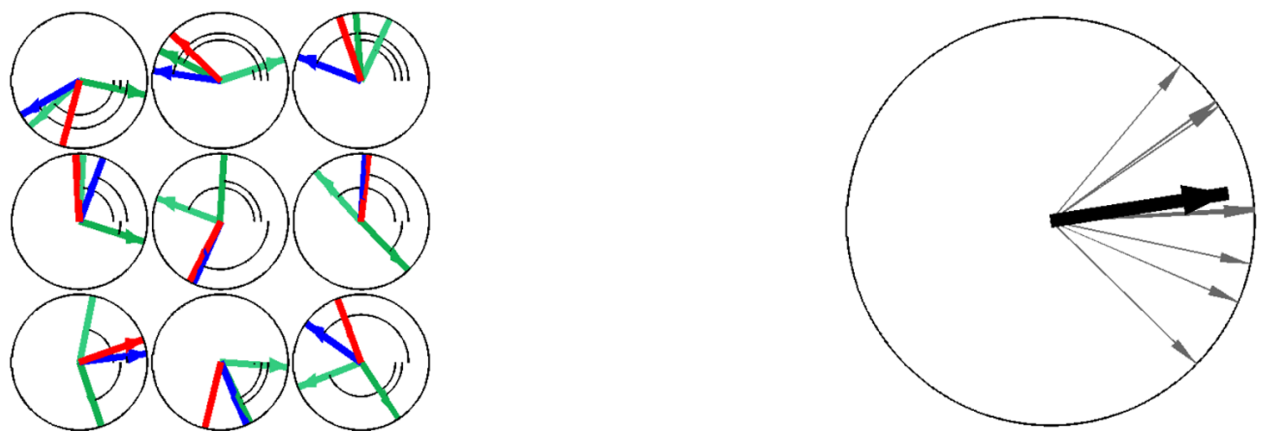

Figure 1. Example of multiplicative frequency coupling. $A, A^{\prime \prime}$ carrier" signal $\tilde{x}\left(t, f_{1}\right)=\exp \left(i\left(2 \pi f_{1} t+\phi_{1}\left(t, f_{1}\right)\right)\right)$ at $80 \mathrm{~Hz}$. The right side shows the time series of the real part of the carrier (thick line) and the instantaneous phase $\phi_{1}\left(t, f_{1}\right)$. The left side shows the associated power spectrum of the real part of the carrier signal. $\boldsymbol{B}$, The $10 \mathrm{~Hz}$ real part of the signal component $\tilde{x}\left(t, f_{2}\right)=\exp \left(i\left(2 \pi f_{2} t\right.\right.$ $\left.\left.+\phi_{2}\left(t, f_{2}\right)\right)\right)$ with instantaneous phase $\phi_{2}\left(t, f_{2}\right)$ (right) and associated power spectrum (left). $\boldsymbol{C}$, Real component of $\exp \left(i\left(2 \pi f_{1} t+\phi_{1}\left(t, f_{1}\right)\right)\right) \exp \left(i\left(2 \pi f_{2} t+\phi_{2}\left(t, f_{2}\right)\right)\right)$, with a phase modulated by the components $\tilde{x}\left(t, f_{1}\right)$ and $\tilde{x}\left(t, f_{2}\right)$ [i.e., $\phi\left(t, f_{1}+f_{2}\right)=\phi_{1}\left(t, f_{1}\right)+\phi_{2}\left(t, f_{2}\right)$ (left) and power spectrum (right), which peaks at $\left.90 \mathrm{~Hz}\right]$. $D$, Trial-wise computation of the bPLV. Left, The green arrows show the phases $\phi_{1}\left(t, f_{1}\right)$ and $\phi_{2}\left(t, f_{2}\right)$ in signal 1 ; the red arrows show the compound phase, $\phi_{1}\left(t, f_{1}\right)+\phi_{2}\left(t, f_{2}\right)$, in signal 1 ; and the blue arrows show the phase at frequency $f_{1}+f_{2}$ in signal 2 . Right, The gray arrows show the trial-wise phase difference between the compound phase in signal 1 and the phase in signal two. The black arrow shows the mean phase difference across trials. The length of the black arrow is the bPLV.

frequency decreases in power that occur with movement (Miller et al., 2007b). Therefore, we focused our analysis on interactions between frequencies ranging from 6 to $24 \mathrm{~Hz}$, covering the alpha and beta bands, with frequencies from 48 to $96 \mathrm{~Hz}$ band (gamma to high gamma).

\section{Materials and Methods}

Subjects. Data were recorded from four epileptic patients who had $8 \times 8$ electrode grid arrays ( $1 \mathrm{~cm}$ interelectrode distance), implanted with sensorimotor coverage, for electrocorticographic localization of seizures. Subjects gave their informed consent according to the protocol approved by the internal review board of the University of Washington. The subject demographics are shown in Table 1.

Tasks. A visual cue was displayed on a liquid crystal display screen at random intervals. Subjects were instructed to move their thumb as soon as possible after the appearance of the visual cue. A total of 30 cues were presented per subject.

Recording. The ECoG data were sampled continuously over the course of the experiment at $1 \mathrm{kHz}$ with an analog low-pass filter of $250 \mathrm{~Hz}$. The exact onset of the movement was recorded by a data glove (5DT Virtual Realities). The data glove output for each finger was also continuously recorded at the same sampling rate.

Data preprocessing. All data were downsampled off-line to $250 \mathrm{~Hz}$ and re-referenced to a common average reference (CAR). A CAR was used because the original references were either scalp or neck references, which were susceptible to line noise and EMG artifacts. We used the increase of the slope of the dataglove output for the thumb to identify the exact movement onset and to segment the ECoG data into trials starting 
A
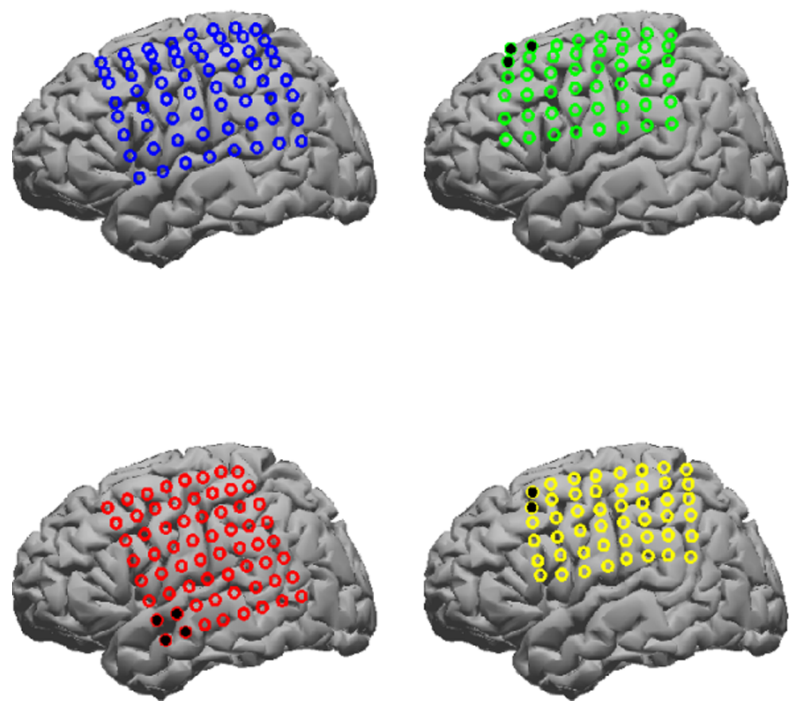

B

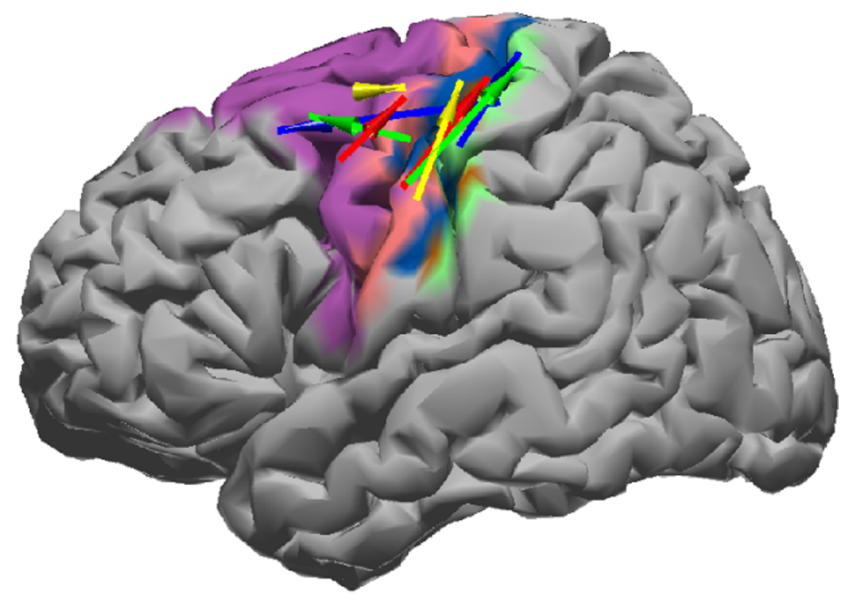

Figure 2. Common phase modulation in the motor cortex across subjects. $\boldsymbol{A}$, Electrode coverage. Blue, Subject 1; green, subject 2; red, subject 3; yellow, subject 4. Note that the positions for subject 1 have been projected from the right to the left hemisphere. The black markers indicate the seizure onset zone as identified by the clinician. $\boldsymbol{B}$, Significant phase-phase interactions were found in all subjects (S1, light blue; $\$ 2$, green; $\$ 3$, red; $S 4$, yellow) after clustering by spatial proximity. Brodmann areas are color coded as follows: BA1, brown; BA2, green; BA3, blue; BA4, pink; BA6, purple. Spatially consistent interactions across subjects form a cluster from premotor sources (BA6) to peri-Rolandic primary motor targets (BA4). Interactions are indicated by color-coded arrows, in which the point of the arrow indicates the direction of interaction.

$-2.5 \mathrm{~s}$ before movement onset and ending at $2.5 \mathrm{~s}$ after movement onset. Only movements after the visual cue were used for segmentation. Visual inspection for artifacts led to the removal of three trials for subject 2; all other subjects were found to be free of artifacts.

Signal analysis. To test for event-related nonlinear relationships between electrode pairs, we used an extension of the standard phaselocking value (PLV) (Tass et al., 1998; Lachaux et al., 1999), the biphaselocking value (bPLV), to analyze the time-resolved phase relationship between two frequency components $\tilde{x}\left(t, f_{1}\right)$ and $\tilde{x}\left(t, f_{2}\right)$ in signal $X(t)$ and the resulting multiplicative component $f_{3}=f_{1}+f_{2}$ in signal $Y(t), \tilde{y}\left(t, f_{3}\right)$. Like the frequencies, the phases of the signals add as well, such that $\phi_{3}(t)=\phi_{1}(t)+\phi_{2}(t)$. The bPLV is related to the bicoherence (Nikias and Mendel, 1993) in the same way as PLV is to coherence. Unlike phase locking, which detects only synchronization of signals in a given band, bPLV can detect modulation of the phase of signal $\tilde{y}\left(t, f_{3}\right)$ at one "target" site by the independent phases of the frequency components $\tilde{x}\left(t, f_{1}\right)$ and $\tilde{x}\left(t, f_{2}\right)$ at a separate "source" site. This can be used as a measure of causality because the uniqueness of the nonlinear relationship between the source site and the target site has definite directionality; the areas are not merely associated, but independent processes in the source area are unambiguously coupled in the target area.

For a number of frequency bins centered at $(6-24 \mathrm{~Hz})$ and $(48-96$ $\mathrm{Hz}$ ), with a width of $6 \mathrm{~Hz}$, we computed the pairwise biphase coupling for each subject. The time-dependent bPLV between three signals $X, Y$, and $Z$ is defined as

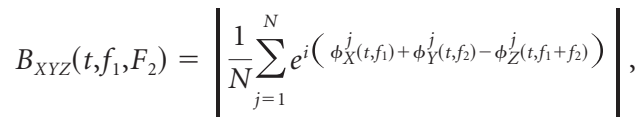

where $\phi_{X}^{j}(t, f)$ is the instantaneous phase of the signal $X(t)$ at frequency $f$ in the $j$ th trial and $N$ is the total number of trials (here, repetitions of the thumb movement). In this study, the instantaneous phases of the signals were computed from the Hilbert transform of the narrow band filtered signals (with the filter centered at $f$ ). We defined the bPLV in the equation above as an interaction between three signals $[X(t), Y(t)$, and $Z(t)]$; however, it can be rewritten as a two-way interaction if we assume that signal $X(t)$ carries independent information in the two frequency bands centered at $f_{1}$ and $f_{2}$. In this case, $B_{X Y Z}$ becomes $B_{X X Y}$, and, because $B_{X X Y}$ $\neq B_{Y X X}$, this measure of interaction between the two signals is a directional measure. Analogous to the conventional PLV, the bPLV takes on values between 0 and 1 , with 0 indicating no phase coupling and 1 indicating perfect phase coupling at these frequencies. In the case of the bPLV, a nonlinearity that produces perfectly biphase locking for a pair of frequencies $\left(f_{1}, f_{2}\right)$ is a multiplication of the respective frequency components [i.e., $\left.\tilde{y}\left(t, f_{1}+f_{2}\right)=\tilde{x}\left(t, f_{1}\right) \tilde{x}\left(t, f_{2}\right)\right]$. The addition of phases and frequencies through multiplication can be easily illustrated by the complex representation of two oscillatory signals at frequencies $f_{1}$ and $f_{2}$ with phases $\phi_{1}$ and $\phi_{2}: \exp \left(i\left(2 \pi f_{1} t+\phi_{1}\right)\right) \exp \left(i\left(2 \pi f_{2} t+\phi_{2}\right)\right)=\exp \left(i\left(2 \pi\left(f_{1}\right.\right.\right.$ $\left.\left.\left.+f_{2}\right) t+\phi_{1}+\phi_{2}\right)\right)$. This relationship is illustrated in Figure 1. The directionality of the bPLV can also be seen directly from the algebraic relationship between the components in $X(t)$ and $Y(t)$ [i.e., $\tilde{y}\left(t, f_{1}+f_{2}\right)=$ $\tilde{x}\left(t, f_{1}\right) \tilde{x}\left(t, f_{2}\right)$ is not necessarily equal to $\tilde{x}\left(t, f_{1}+f_{2}\right)=\tilde{y}\left(t, f_{1}\right) \tilde{y}\left(t, f_{2}\right)$ unlike in classical coherence, where for two perfectly coherent components $\tilde{x}(t$, $\left.f_{1}\right)=\tilde{y}\left(t, f_{1}\right)$ holds and the order of $X(t)$ and $Y(t)$ is of no relevance]. It should also be noted, that although this measure is directional, it is not necessarily a measure of causality, unless the components $\tilde{x}\left(t, f_{1}\right)$ and $\tilde{x}\left(t, f_{2}\right)$ in signal $X(t)$ are independent. If in signal $X(t)$ the phases $\phi_{1}$ and $\phi_{2}$ are independent, then we have causality from $X(t)$ to $Y(t)$, because both phases $\phi_{1}$ and $\phi_{2}$ have to be known to predict $\phi_{3}=g\left(\phi_{1}, \phi_{2}\right)=\phi_{1}+\phi_{2}$ in $Y(t)$. Conversely, knowledge of just $\phi_{3}$ cannot be used to predict $\phi_{1}$ and $\phi_{2}$.

If $\phi_{2}=\phi_{2}\left(\phi_{1}\right)$ (i.e., if there is dependence of $\phi_{2}$ on $\phi_{1}$ ), then it follows that $\phi_{3}=g\left(\phi_{1}\right)$ [i.e., $\phi_{3}$ is only dependent on $\phi_{1}$, and consequently $\phi_{1}=$ $\left.g^{-1}\left(\phi_{3}\right)\right]$ (i.e., $\phi_{1}$ could be predicted equally well from $\phi_{3}$ as $\phi_{3}$ from $\phi_{1}$ ). In this case, the bPLV would not be a directional measure.

Effect of the common average reference on the bPLV. Because a CAR was subtracted from the data before additional processing, the bPLV was computed between signals $\tilde{y}\left(t, f_{1}+f_{2}\right)=\tilde{y}^{*}\left(t, f_{1}+f_{2}\right)-\tilde{c}\left(t, f_{1}+f_{2}\right), \tilde{x}(t$, $\left.f_{1}\right)=\tilde{x}^{*}\left(t, f_{1}\right)-\tilde{c}\left(t, f_{1}\right)$, and $\tilde{x}\left(t, f_{2}\right)=\tilde{x}^{*}\left(t, f_{2}\right)-\tilde{c}\left(t, f_{2}\right)$, where ${ }^{*}$ indicates the "true" signals and $\tilde{c}(t, f)$ are the frequency components of the CAR. An observation of a high bPLV between signals $X(t)$ and $Y(t)$ thus could be attributable to biphase coupling in the CAR instead of being a genuine effect between the true signals, similar to apparent increases in coherence between channels because of power changes in the CAR. However, be- 
A

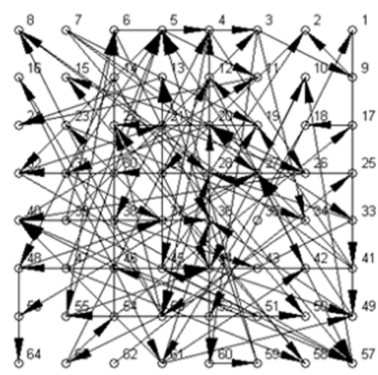

B

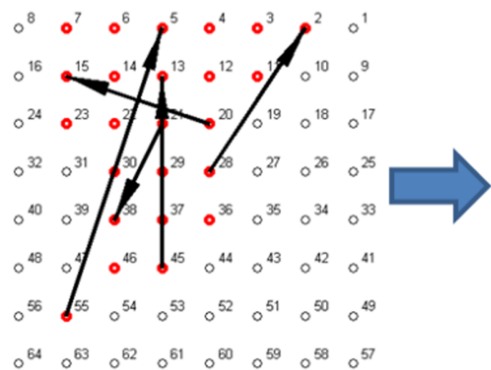

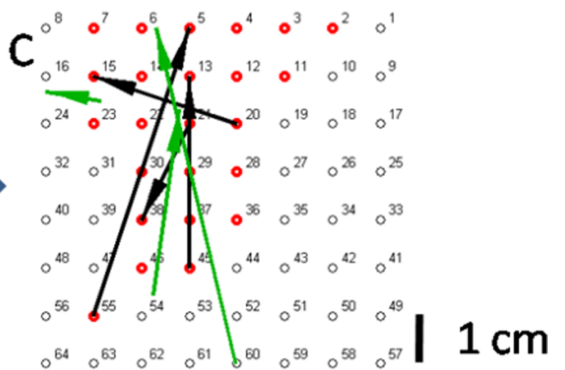

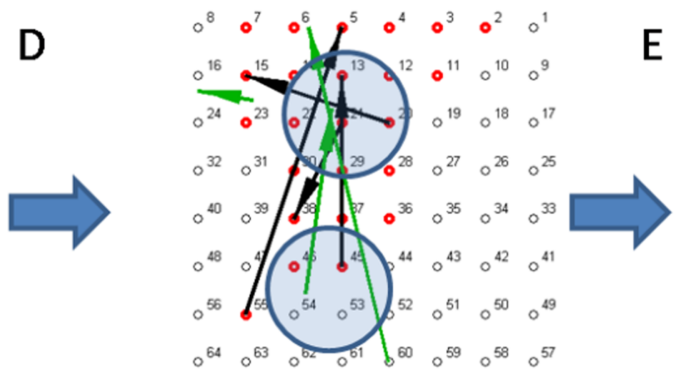

$\begin{array}{llllllll}0^{8} & 0^{7} & 0^{6} & 0^{5} & 0^{4} & 0^{3} & 0^{2} & 0^{1}\end{array}$

$0^{16} 0^{15} \circ^{14} 0^{13} 0^{12} 0^{11} 0^{10} 0^{9}$

$\begin{array}{lllllllll}0^{24} & 0^{23} & 0^{22} & \mathbf{l l l l l}^{21} & 0^{20} & 0^{19} & 0^{18} & 0^{17}\end{array}$

$\begin{array}{llllllllll}0^{32} & 0^{31} & 0^{30} & f^{29} & 0^{28} & 0^{27} & 0^{26} & 0^{25}\end{array}$

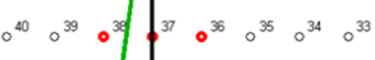

$\begin{array}{lllllllll}0^{48} & 0^{47} & \text { of } & 0^{45} & 0^{44} & 0^{43} & 0^{42} & 0^{41}\end{array}$

$\begin{array}{llllllll}0^{56} & 0^{55} & 0^{54} & 0^{53} & 0^{52} & 0^{51} & 0^{50} & 0^{49}\end{array}$

$\begin{array}{lllllllll}0^{64} & 0^{63} & 0^{62} & 0^{61} & 0^{60} & 0^{59} & 0^{58} & 0^{57}\end{array}$

Figure 3. Clustering scheme to find spatially consistent interactions across subjects. $A$, Initially, a large number of interactions between grid electrodes with uncorrected significances $(p<0.05)$ is found for each subject. $\boldsymbol{B}$. Interactions are restricted to electrodes that lie in Brodmann areas 1,2,3,4, and 6 (red circles). $\boldsymbol{C}$. This procedure is applied to each subject. Exemplary, we show interactions from two subjects (black and green) overlaid on the same grid. Note that the individual grids can have different electrode numbering schemes, and thus electrodes with different indices can cover different Brodmann areas in different subjects. $\boldsymbol{D}$, Interactions are grouped into clusters by spatial proximity. Interactions for which the beginning and end points are within a $2.8 \mathrm{~cm}$ range (the farthest distance between two electrodes in an 8-electrode neighborhood), indicated by the transparent blue circles, and which cover a distance $>2.8 \mathrm{~cm}$ are grouped into the same cluster. $\boldsymbol{E}$, The final cluster configuration.

Table 2. Mean increase in bPLV during movement and premovement

\begin{tabular}{lllll}
\hline Subject & $\begin{array}{l}\text { Interaction } \\
\text { (premotor } \rightarrow \text { motor) }\end{array}$ & $\begin{array}{l}\mathrm{bPLV} \text { value, } \\
\text { rest }\end{array}$ & $\begin{array}{l}\mathrm{bPLV} \text { value, } \\
\text { movement }\end{array}$ & $\begin{array}{l}p \text { value, } \\
\text { movement (corrected) }\end{array}$ \\
\hline 1 & $(12,60) \mathrm{Hz}$ & 0.16 & 0.25 & 0.0337 \\
2 & $(12,78) \mathrm{Hz}$ & 0.14 & 0.29 & 0.0339 \\
3 & $(24,54) \mathrm{Hz}$ & 0.17 & 0.25 & 0.0357 \\
4 & $(6,48) \mathrm{Hz}$ & 0.16 & 0.26 & 0.0077 \\
\hline
\end{tabular}

cause the CAR component is the same in each channel, a purely CARinduced bPLV would manifest itself as a symmetric interaction across channels (i.e., we would expect $\tilde{B}_{X X Y}=\tilde{B}_{Y X X}$ ). Conversely, nonsymmetric interactions can only arise from interactions between parts of the signal that are not common across channels.

Scanning. Because we had no a priori knowledge about specific frequency pairs and interaction sites, we tested all electrode pairs and a range of low-to-high frequency pairs for movement-specific increase of bPLV. To separate increases in bPLV caused by genuine cortical interactions from spurious changes of the bPLV, we computed the significance of the movement-related bPLV increase with respect to the baseline distribution of bPLV values during a rest period before movement. The baseline distribution, which was used as null hypothesis, was estimated by a permutation of signal segments in the baseline. Values of $p$ for the increase of the bPLV above the baseline were Bonferroni corrected for multiple comparisons across channel pairs and frequencies.

For each channel pair $(X, Y)$ of the 4096 (S1 and S3, for which 64 channels from the grid were recorded) or 2304 (S2 and S4, for which 48 channels were recorded) possible pairs, $X$ was bandpass filtered with the band center at $f_{1}$ and $f_{2}$ and a bandwidth of $6 \mathrm{~Hz}$, and $Y$ was filtered at a band center $f_{1}+f_{2}$. Unlike for symmetric measures like coherence or PLV, we have to consider all $n^{2}$ pair combinations for $n$ channels, because of the directionality of the measure. The bPLV from channel 1 to channel 2 is not equal to bPLV from channel 2 to channel 1. It should also be noted that this measure allows for interaction of a channel with itself because independent information can be contained in the two frequency bands at $f_{1}$ and $f_{2}$ in a single channel. We stepped through the bands in 6 $\mathrm{Hz}$ steps from 6 to $24 \mathrm{~Hz}$ for the low-frequency bands and from 48 to 96
$\mathrm{Hz}$ for the high-frequency bands, resulting in a total of 36 frequency bins, and computed the time-resolved bPLV for each channel and frequency pair. The result of this analysis for each channel and frequency pair is a set of $n^{2} x$ number of frequency bins time series, each representing the temporal evolution of the bPLV from $-2.5 \mathrm{~s}$ before movement onset to $2.5 \mathrm{~s}$ after movement onset $(147,456$ time series for S1 and S3, 82,944 time series for S2 and S4) and each time series consisting of 1249 samples. Because we have no a priori knowledge about the specific temporal evolution of the bPLV for the given paradigm and to reduce the amount of data to be tested, we integrated the time series for each pair and frequency bin over time. To test the hypothesis, whether there is a movement-related increase in bPLV, we computed the temporal mean of the bPLV over the movement interval from 0 to $1 \mathrm{~s}$, with $0 \mathrm{~s}$ at the beginning of the movement, and compared it with the temporal mean bPLV over the premovement interval from -1.5 to $-0.5 \mathrm{~s}$.

Statistical testing. To test our hypothesis, that the mean bPLV in the movement interval is increased compared with the mean bPLV in the premovement or rest interval, we have to learn the null distribution of this statistic under the rest condition. Given this distribution, we can compute $p$ values for the mean bPLV during movement. Because we have only $27-30$ trials per subject and the bPLV is defined as a mean quantity over these trials (i.e., we just have a single sample of the bPLV per subject for the movement segment) (i.e., [0 s $1 \mathrm{~s}]$ and the premovement segment $[-1.5 \mathrm{~s}-0.5 \mathrm{~s}])$, we have to use resampling techniques to get the null distribution of the mean bPLV under rest condition.

We used a permutation test to estimate the distribution of the mean bPLV for the rest period that was free from any finger movement. We randomly exchanged the $[-1.5 \mathrm{~s}-0.5 \mathrm{~s}]$ segment with the $[-2.5 \mathrm{~s}-1.5$ s] segment, which was also movement free, in a subset of the thumb movement trials. Our assumption in this permutation scheme is that the underlying distribution of the mean bPLV is the same for both segments, and thus by permuting segments within the baseline we can get an estimate of that baseline distribution. In traditional permutation tests, the permutation is often performed between baseline and signal to learn the 
null distribution. Intervals in a permutation test have to be exchangeable under the null hypothesis (Pantazis et al., 2005). However, because we are actually assuming that our null hypothesis does not hold during movement in the actual data, in which we assume that the mean bPLV is higher than during the rest period, permuting signal and baseline segments would bias the resulting null distribution toward higher bPLV values and thus lead to artificially lower $p$ values for the movement mean bPLV. Therefore, permuting segments in the baseline is the better choice to learn the null distribution, because we are not contaminating our permutation samples with segments in which the mean bPLV is high because of movement, and are thus increasing the power of our statistical test (Efron et al., 2001; Chau et al., 2004).

We repeated this permutation 1000 times to estimate reliably the distribution of the mean bPLV under rest condition for each channel and frequency pair. The bPLV is bounded between 0 and 1 , and we can approximate its probability density function by a bounded analytical distribution. We chose the twoparameter $\beta$ distribution, which can approximate the empirical bPLV distribution well, to compute the $p$ values for bPLV during movement. This analytic approximation is useful, because we have to correct the $p$ values for the total number of channel pairs under consideration, and this would normally require a very large number of permutations $\left(\sim 10^{5}\right)$, which would lead to prohibitively long computation times.

Selection of consistent interactions across subjects. To find interactions among the larger number of possible interactions, which are common and significant across all subjects, we apply a number of spatial and statistical filters to our data.

Individual channel positions were mapped into a standard Talairach atlas, based on x-ray images. For better comparability, we projected positions for the right hemisphere subject (subject 1 ) also onto the left hemisphere. The individual montages are shown in Figure $2 \mathrm{~A}$. Our initial hypothesis is that, during movement, bPLV is increased in specific channel pairs in the sensorimotor system. Therefore restrict our choice of interaction pairs (from a total of $82,944-147,456$ ) to those that originate and end in the sensorimotor areas [i.e., the somatosensory, premotor, and motor areas (Brodmann areas 1, 2, 3, 4, and 6)]. We identified these Brodmann areas in Talairach space, and, because we mapped all our individual electrode positions into that space, we can determine for each electrode and subject in which functional area it lies. This restriction is motivated by the experimental paradigm (i.e., the finger movement task), in which we naively expect a task-related response in these areas. We computed $p$ values for each interaction pair in the sensorimotor area and we corrected them for multiple comparisons across all possible channel pairs in these areas and for the number of frequency bins, using a conservative Bonferroni correction. We found 576 pairs in S1, 484 in S2, 400 in S3, and 484 in S4. With a total of 36 frequency pair bins, this results in a correction for 20,736 tests in S1, 17,424 tests in S2, 14,400 tests in S3, and 17,424 in S4. To select only significant interactions for additional analysis, we chose from all channel pairs those interactions with a corrected value of $p<$ 0.05 . From these, a subset of interactions was selected, which exhibited the greatest spatial coherence across all subjects (i.e., interactions in which the corresponding electrode positions for the start and endpoint of interaction were $<2.8 \mathrm{~cm}$ separated in the common Talairach brain atlas). If interactions were on the same grid, $2.8 \mathrm{~cm}$ is the maximum dis- tance between two electrodes that are separated by just one grid position between them. This selection criterion is based on the assumption that functionally similar processes should lie in similar anatomical region across subjects. To select long-range interactions, we further limited the selection to interactions that covered a distance of $>2.8 \mathrm{~cm}$. This additional selection ensures also that we select only interactions that can be reliably identified as spatially consistent, given the overall low spatial resolution of the electrode placement. Also, potential volume conduction effects between electrodes, that are further apart, can be expected to be small (Lachaux et al., 1999). A graphical example of the selection process is shown in Figure 3.

\section{Results}

\section{Data-driven interactions}

Across all four subjects, we found 6 interactions in S1 with a corrected value of $p<0.05$ in the sensorimotor region, 3 interactions in S2, 10 interactions in S3, and 5 interactions in S4. Spatial clustering of these results, as described in the previous section, results in a single, spatial consistent cluster of interactions across all subjects between premotor "sources" (BA6) to peri-Rolandic primary motor "targets" (BA4). This cluster of interactions contains a single interaction per subject, which we label by the anatomical direction of interaction (i.e., premotor $\rightarrow$ motor) and the frequencies across which these electrodes couple [e.g., $(12,60) \mathrm{Hz}$ premotor $\rightarrow$ motor in S1]. A mapping of these 


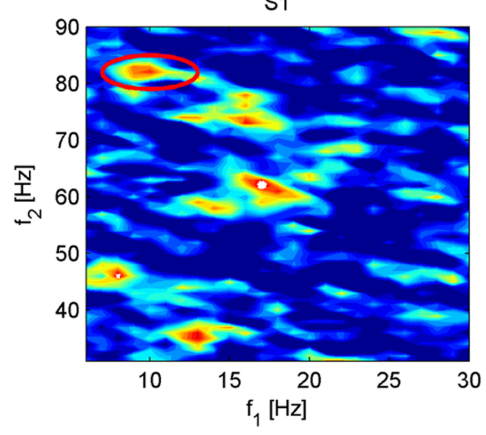

S3

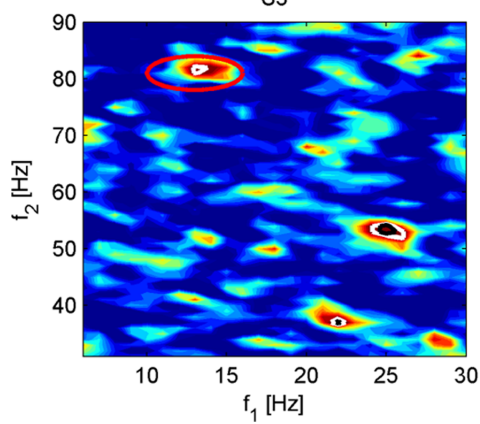

S2

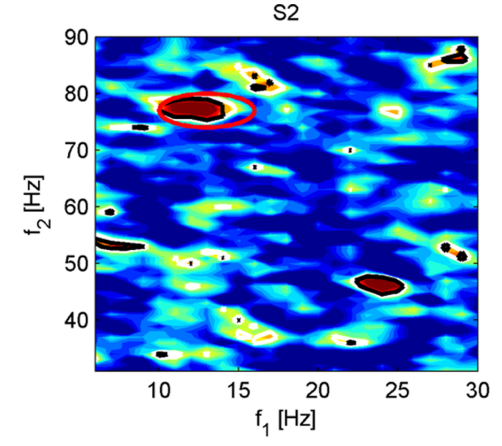

S4

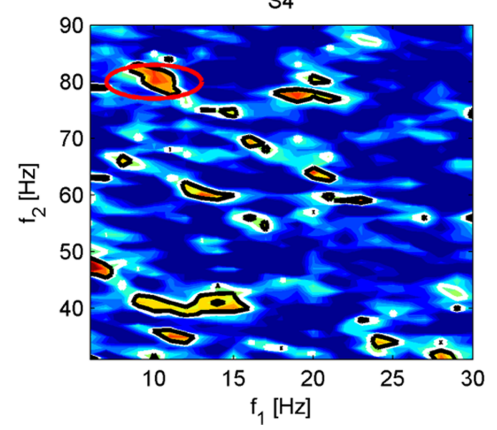

Figure 5. Frequency-frequency maps of the mean bPLV over the movement segment $[0 \mathrm{~s}-1 \mathrm{~s}]$ for channel interaction between premotor-to-motor channel pairs for all subjects. Note that there is a strong similarity for $\mathrm{S} 2$ and $\mathrm{S} 3$ in these maps. The common interaction frequency located at approximately $(10,80 \mathrm{~Hz})$ is indicated by the white circles. The Bonferroni-corrected significance levels, in which we correct for the number of frequency bins, are indicated by the white ( $p \leq 0.2)$ and black ( $p \leq$ $0.05)$ contour lines.

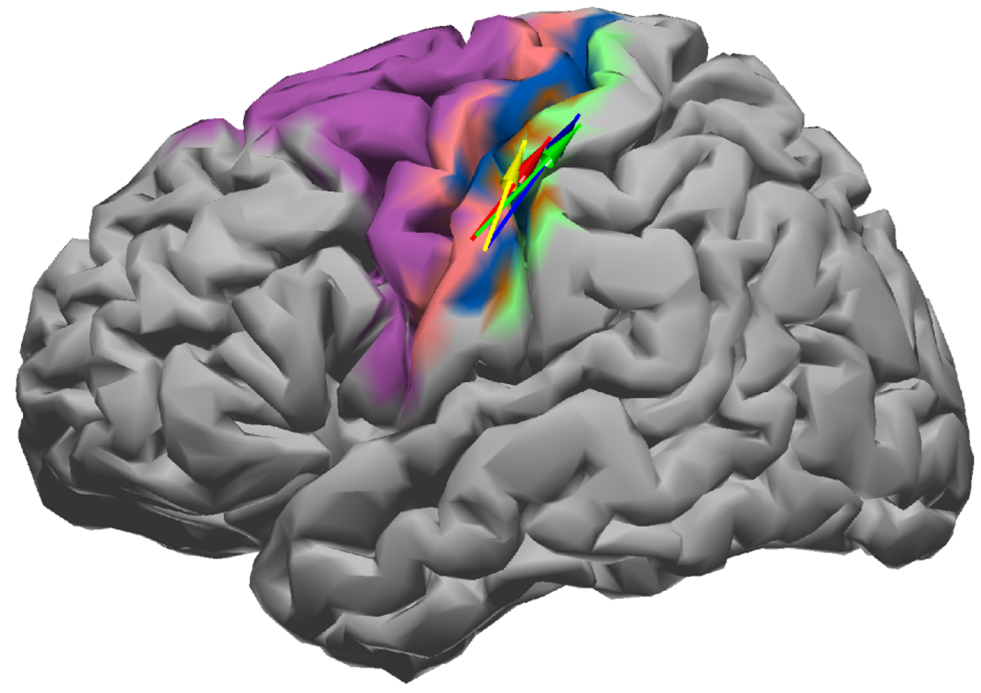

Figure 6. Interaction cluster with revised position for the interaction in S1. The same color coding as in Figure 2 is used. The mean Talairach coordinates across all subjects were at $x=-58, y=-14, z=39$ (source site), and $x=-38, y=-31, z=$ 63 (target site).

interactions onto the generic cortical surface is shown in Figure $2 \mathrm{~B}$. At a ventral premotor source site, we find low frequencies of 6,12 , and $24 \mathrm{~Hz}$ interacting with $48,60,78$, and $54 \mathrm{~Hz}$ to produce the coupled frequency in the dorsal target site over motor cortex. In all subjects, seizure foci (Table 1) were well away from the areas of interest we found and all patients were studied during a period of relative epileptic silence. Stimulation mapping for all subjects also confirmed that they had motor evoked responses at expected positions and currents. The bPLV interactions we found were just one grid position posterior to these mappings.

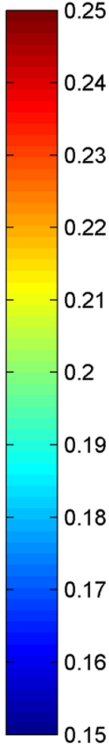

In Table 2, we show the mean bPLV during movement for these interactions along with the mean bPLV during rest, along with the corrected $p$ values for these increases. In Figure 4, we show the temporal evolution for the bPLV value from $-2.5 \mathrm{~s}$ before movement onset to $2.5 \mathrm{~s}$ past movement onset along with the output of the data glove. In all subjects, there is a significant increase in bPLV for these channel pairs, which coincides with the actual movement onset as recorded by the data glove.

\section{Test for external stimulus \\ locked influences}

To ensure that these increases are not caused by stimulus or motor-locked artifacts, we used an additional permutation scheme, in which we now permute the trial order between interacting pairs of channels, before computing the mean bPLV for the movement and the rest period (Lachaux et al., 1999) (i.e., we test whether channel $x$ in the $i$ th trial is biphase locked with channel $y$ in the $j$ th trial). The rationale for this test is to reveal whether the observed interaction is attributable to some external fixed stimulus locked event (e.g., the movement of the data glove that would be independent of the trial order) or caused by genuine cortical interactions. Stimulus locked events will be invariant under permutation of the trial order and if the increase in bPLV between two channels is caused by such events, it can be expected to remain high, when we shuffle the trial order for one of the channels. We generated 10,000 permutation samples of the mean bPLV for each subject and determined the $p$ value for our measured values of the mean bPLV as the proportion of resampled values that exceed the original value. We applied this permutation test to the rest and the movement period. The resulting $p$ values for all subjects for this set of interactions are shown in Table 3. For each subject, the bPLV increase during movement is highly sensitive to the specific trial order and as such to the precise timing within each trial. In contrast, we find that the premovement period is not affected by trial shuffling, which confirms that there is no bPLV in the rest period before the finger movement.

\section{Post hoc analysis}

Having demonstrated significant interactions of given frequency bands for specific electrodes, we examined post hoc the interactions between these cortical regions across all low-to-high frequency couplings. This post hoc analysis is motivated by the conservative threshold that we applied in our initial search for bPLV 
interactions and our initial coarse frequency band spacing, which could have obscured frequency and channel pairs, that could have shown up as significant in a hypothesis-driven approach. We computed the mean bPLV increase during movement over a broader frequency range from 6 to $30 \mathrm{~Hz}$ over 31 to $90 \mathrm{~Hz}$ in $1 \mathrm{~Hz}$ steps for all channel pairs in the sensorimotor region for each subject to identify common channel and frequency pairs across all subjects. We used the initial channel pairs found by our datadriven approach to test for the hypothesis, whether there are interactions between similar anatomical locations involving similar frequencies. Figure 5 shows the frequency maps for the channel pairs that we identified in our previous analysis for subjects S2-S4. These maps show the strongest increase in the mean bPLV during movement near the frequencies that we previously identified. Based on these maps, we find that the strongest interaction for S2 between premotor and motor is not located at $(12,78) \mathrm{Hz}$ but at $(13,77) \mathrm{Hz}$. However, we also find that, in S3, there is a strong increase in bPLV between premotor and motor at frequencies $(13,81) \mathrm{Hz}$ in addition to the previously found pair at $(24,54) \mathrm{Hz}$. Likewise, we find for S4 an additional interaction at $(10,80) \mathrm{Hz}$. Although we did not find a peak near $(10$, 80) $\mathrm{Hz}$ in the map for the initial premotor $\rightarrow$ motor channel pair in S1, inspection of the maps for neighboring channel pairs showed that the lower left neighbor of the motor channel in the montage for S1 has increased mean bPLV during movement at frequencies $(10,82) \mathrm{Hz}$ (Fig. 5). The interaction pairs $(10,82)$ $\mathrm{Hz}$ motor $\rightarrow$ premotor for $\mathrm{S} 1,(13,77)$ $\mathrm{Hz}$ for S2, $(13,81) \mathrm{Hz}$ for S3, and $(10,80)$ $\mathrm{Hz}$ for $\mathrm{S} 4$ along with the respective Talairach coordinates are shown in Figure 6.

The mean bPLV increase during movement versus the mean bPLV increase during rest as well as the $p$ values for the movement-related increase are shown in Table 4 . Note that we show uncorrected $p$ values for these interactions. The time courses of the bPLV for these interactions are shown in Figure 7. If we test for the general hypothesis that any channel pair at any frequency during finger movement shows increased bPLV, these interactions will not be significant because of the multiple comparison correction and our data-driven analysis initially did not reveal those interactions. However, when we test for the specific hypothesis that these channel pairs interact at the given frequencies, we find them to be highly significant $(p<0.01$, post hoc) across all four subjects. Furthermore, these interactions are consistent in frequencies and anatomical location. It is also noteworthy that we find for $\mathrm{S} 1$, who carried out left-handed thumb movements and where recordings were made from the right hemisphere, the same bPLV increase was observed as for the
Table 4. Mean increase in bPLV during movement and premovement

\begin{tabular}{llllc}
\hline Subject & Interaction (premotor $\rightarrow$ motor) & bPLV value, rest & bPLV value, movement & $p$ value, movement \\
\hline 1 & $(10,82) \mathrm{Hz}$ & 0.19 & 0.24 & 0.0123 \\
2 & $(13,77) \mathrm{Hz}$ & 0.14 & 0.28 & $<0.00001$ \\
3 & $(13,81) \mathrm{Hz}$ & 0.14 & 0.26 & 0.0044 \\
4 & $(10,80) \mathrm{Hz}$ & 0.14 & 0.24 & $<0.00001$ \\
\hline
\end{tabular}
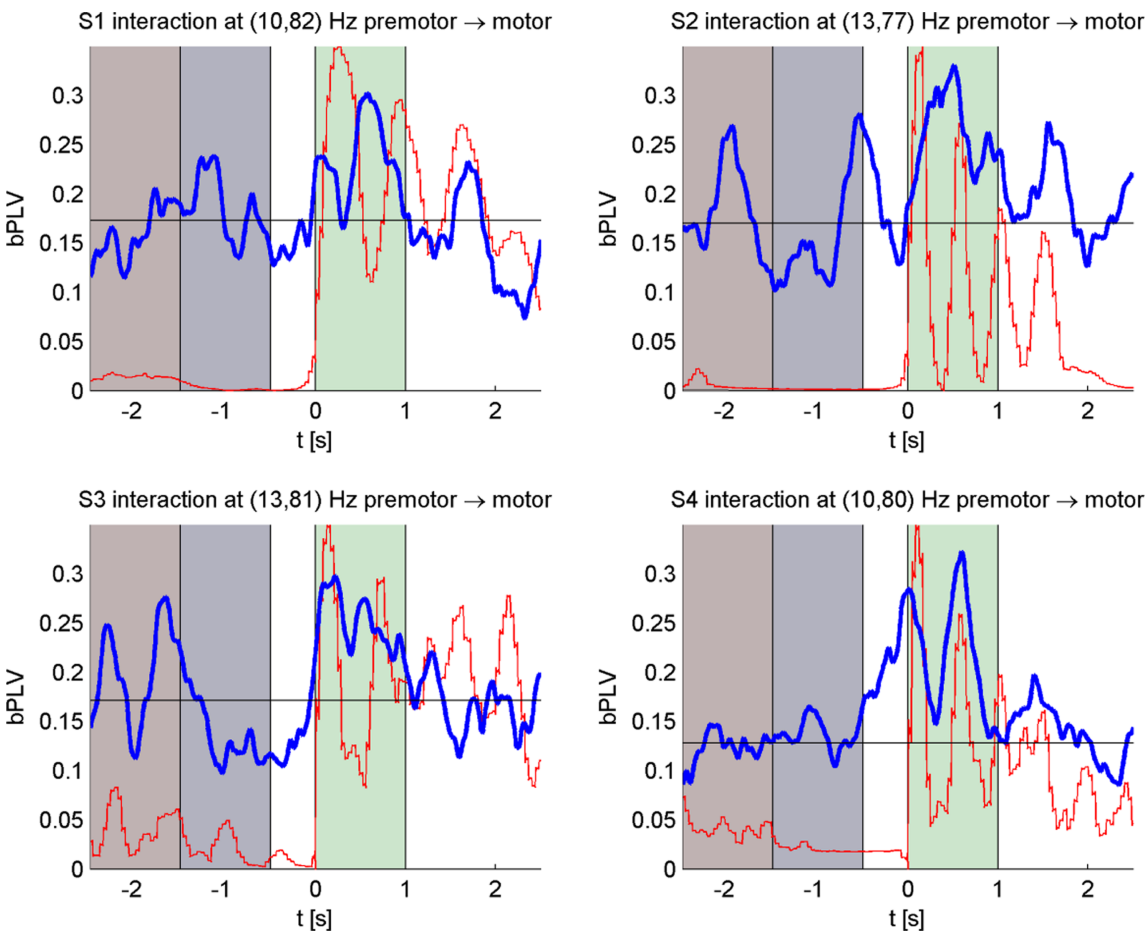

Figure 7. Temporal evolution of the bPLV for selected premotor-to-motor channel pairs.

Table 5. p values for trial shuffling for post hoc analysis

\begin{tabular}{llll}
\hline Subject & Interaction & $\begin{array}{l}p \text { value, } \\
\text { rest }\end{array}$ & $\begin{array}{l}p \text { value, } \\
\text { movement }\end{array}$ \\
\hline 1 & $(10,82)$ Hz premotor $\rightarrow$ motor & 0.1306 & 0.0030 \\
2 & $(13,77)$ Hz premotor $\rightarrow$ motor & 0.6389 & 0.0002 \\
3 & $(1381)$ Hz premotor $\rightarrow$ motor & 0.6243 & 0.0007 \\
4 & $(10,80)$ Hz premotor $\rightarrow$ motor & 0.8953 & 0.0036 \\
\hline
\end{tabular}
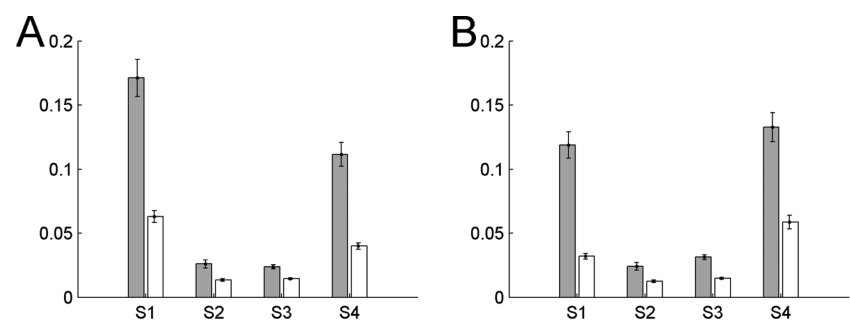

Figure 8. Mean mutual information (over trials) for each subject between the phases $\phi_{1}\left(t, f_{1}\right)$ and $\phi_{2}\left(t, f_{2}\right)$ (white bars) in the premotor region during movement and mean mutual information between the phase in the motor region, $\phi\left(t, f_{1}+f_{2}\right)$, and the sum of the phases in the premotor region (gray), $\phi_{1}\left(t, f_{1}\right)+\phi_{2}\left(t, f_{2}\right)$, during movement. The error bars indicate the SEM. $A$ shows the mutual information for the results from the initial analysis, and $\boldsymbol{B}$, for the post hoc results.

other three subjects with left hemispheric coverage. This indicates that this effect is not tied to a specific hemisphere. To test for external stimulus locked events, we applied the trial shuffling permutation tests as previously described. The resulting $p$ values 
$\mathrm{S} 1 \alpha$ band $7-13 \mathrm{~Hz}$

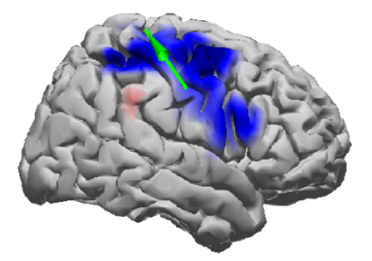

$\mathrm{S} 2 \alpha$ band $10-16 \mathrm{~Hz}$

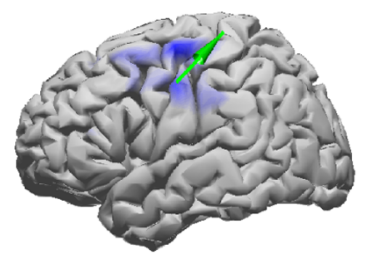

$\mathrm{S} 3 \alpha$ band $10-16 \mathrm{~Hz}$

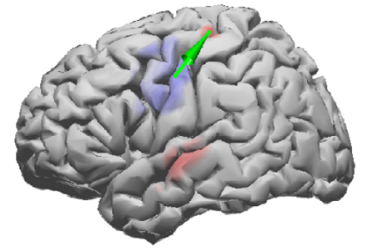

S4 $\alpha$ band $7-13 \mathrm{~Hz}$

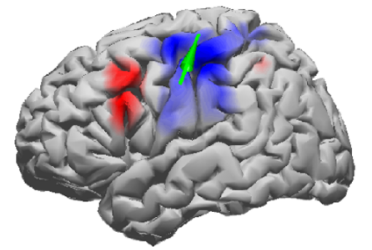

S1 mid- $\gamma$ band $79-85 \mathrm{~Hz}$

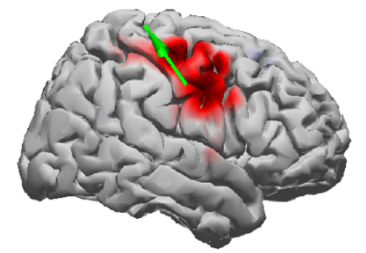

S2 mid- $\gamma$ band $74-80 \mathrm{~Hz}$

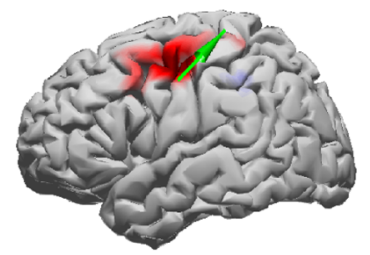

S3 mid- $\gamma$ band $78-84 \mathrm{~Hz}$

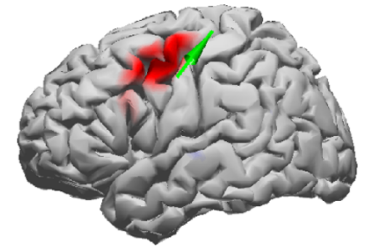

S4 mid- $\gamma$ band $77-83 \mathrm{~Hz}$

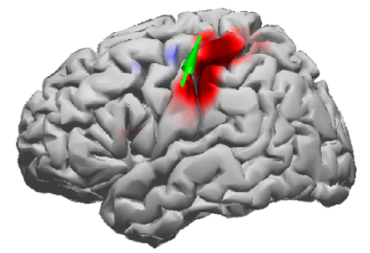

S1 high- $\gamma$ band $89-95 \mathrm{~Hz}$

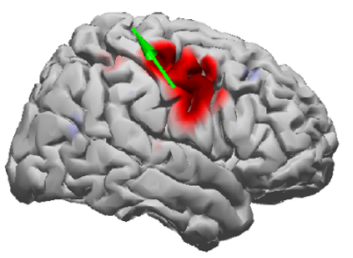

S2 high- $\gamma$ band $87-93 \mathrm{~Hz}$

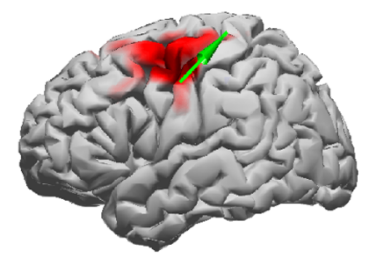

S3 high- $\gamma$ band $91-97 \mathrm{~Hz}$

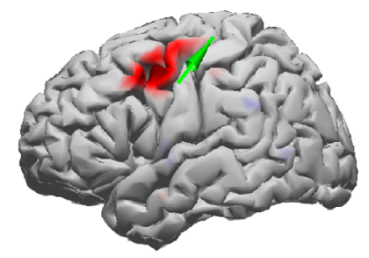

S4 high-y band $87-93 \mathrm{~Hz}$

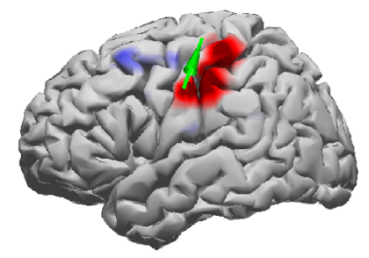

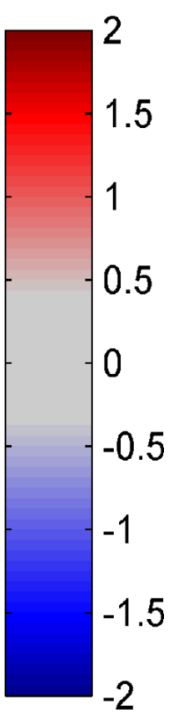

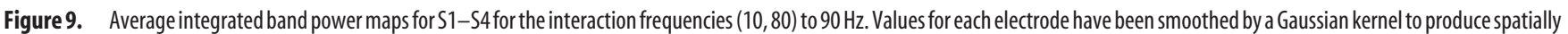
smooth maps (Miller et al., 2007a). The band power for each band has been integrated over the movement time period [ $0 \mathrm{~s}-1 \mathrm{~s}$ ] and normalized with respect to the baseline period [ $-1.5 \mathrm{~s}$ to -0.5 s]. The green arrows indicate the nonlinear interaction for premotor-to-motor electrode pairs in S1, S2, S3, and S4. The frequency bands were selected based on the interacting cross-frequencies as identified by the $\mathrm{pBLV}$ increase during movement.

are shown in Table 5. Similar to our previous analysis, we find that the bPLV during movement is highly sensitive to the trial order, whereas the rest period bPLV is not, which indicates that the increase in bPLV that is observed during movement for these channel and frequency pairs is not caused by stimulus locked external events.

\section{Independence of the phases in the premotor region}

To test for the directionality of the observed bPLV, we analyze the dependence of the phases $\phi_{1}$ and $\phi_{2}$ in the premotor region during movement (i.e., in the interval from $\left[\begin{array}{lll}0 & \mathrm{l} & \mathrm{s}\end{array}\right]$ ). We computed the mutual information for each trial and subject between the phases $\phi_{1}$ and $\phi_{2}$ of the signal in the premotor region during movement and compare this with the mutual information of the sum of these phases [i.e., $\phi_{1}\left(t, f_{1}\right)+\phi_{2}\left(t, f_{2}\right)$ to the respective phase in the motor region $\left.\phi\left(t, f_{1}+f_{2}\right)\right]$. The results for each subject are shown in Figure 8. In all subjects, the mean mutual information between the phases in the premotor region is considerably lower than the mutual information between the sum of these phases and the respective phase in the motor region. This means that $\phi_{1}$ and $\phi_{2}$ are less mutually dependent than $\phi_{1}\left(t, f_{1}\right)+$ $\phi_{2}\left(t, f_{2}\right)$ and $\phi\left(t, f_{1}+f_{2}\right)$, which shows that the measured bPLV is indeed a directional measure from the premotor region to the motor region.

Power changes and coherence changes in the involved frequency bands

To get a better understanding of the processes that lead to increase in bPLV during movement, we analyze the power, coherence, and PLV changes between movement and premovement period for all subjects. We focus on the common frequency pair interactions [i.e., the $(10-13,77-82) \mathrm{Hz} \rightarrow(90-94) \mathrm{Hz}$ interactions], because these are the more interesting candidates for a common interaction mechanism. The power changes for these bands (i.e., 10-13, 77-82, and 90-94 Hz) are shown in Figure 9, in which the average integrated band power increase during movement $[0 \mathrm{~s} \quad 1 \mathrm{~s}]$ over the baseline $[-1.5 \mathrm{~s}-0.5 \mathrm{~s}]$ in the respective band is shown for each subject for all electrodes. We used the mean and SD of the respective band power over trials for the baseline to convert the band power during movement into 
normalized $Z$ scores (i.e., the scale on the maps is given in SDs of the baseline band power). A detailed analysis of the power changes for these subjects during hand and finger movement can also be found in the studies by Miller et al. (2007a,b). What is noteworthy here is the fact that the channels involved in the bPLV interaction (indicated by the green arrows) do not coincide with the channels that show the most relevant power increases/decreases in the involved bands. This indicates that the observed bPLV coupling is not correlated with movement-specific power changes, but is indeed a truly phase-related phenomenon. Also, we did not find any significant increases in bPLV over the baseline at the sites of the strongest power increases/ decreases. The results of the premovement/movement coherence and phaselocking analysis are shown in Tables 6 and 7.

We applied the same baseline-based permutation test for both measures, coherence and phase locking, to determine the significance of the movement-related increases/decreases. Because the selection of interacting channels and frequencies is hypothesis driven, we have only a single comparison per subject and thus do not correct our $p$ values. For individual subjects, we find significant changes in the coherence/ phase-locking value during movement; however, these are not consistent across subjects, unlike what we find for the bPLV increase. For S1, we find significant decreases of the coherence for the low-frequency band (with center at $10 \mathrm{~Hz}$ ) and the highfrequency band at the coupled frequency (with center at $92 \mathrm{~Hz}$ ), whereas this high-frequency decrease for the PLV is not significant, which indicates that the interactions for these bands for this subject are mostly amplitude driven. Overall, we do not find consistent changes in the coherence and PLV for all subjects (i.e., no consistent synchronization or desynchronization in the bands involved in the consistent bPLV changes).

\section{Discussion}

We have shown consistent thumb movement-related increase in bPLV between the premotor and primary motor area in four subjects. The interaction is temporally locked to the onset of thumb movement and is unidirectional, from premotor to primary motor cortex. This demonstrates the existence of taskspecific, distributed, and nonlinear networks in the human motor system, activated during movement. These motor networks involve phase-phase coupling across frequencies, which results from a multiplicative interaction. These interactions occur at specific frequencies $(10-13,77-82) \mathrm{Hz}$ across all subjects. This suggests that there are common specific frequencies that play a role during movement, similarly to the alpha-beta suppression during movement (Crone et al., 1998) but may manifest as subject-specific frequencies, perhaps depending on specific aspects of the individual movement execution. The individually most significant frequencies cover a wide range across the alphabeta spectrum, as do the frequencies with which they interact. The low components of the common frequencies that interact fall all within the alpha band $(10-13 \mathrm{~Hz})$ and likewise the high frequencies range only from 77 to $82 \mathrm{~Hz}$, as well the resulting frequencies $(90-94 \mathrm{~Hz})$. Furthermore, for the $(10-13,77-82) \mathrm{Hz}$ interaction, we do not find consistent phase synchronization, coherence, or amplitude changes across all subjects between the involved anatomical regions. This shows, on one hand, that the observed task-related bPLV increase during movement is not an epiphenomenon of an underlying single band synchronization or amplitude effect, and, on the other hand, because theoretically single-band synchronization and across-band synchronization can coexist, that the cross-frequency coupling demonstrated here is an exclusively nonlinear effect. Furthermore, the dissociation between areas involved in this nonlinear coupling and areas of single band changes allow speculation that this coupling provides information transfer between brain regions not obtainable from simple synchronization and/or power amplitude changes.

A possible role of these (alpha, gamma) to higher gamma interactions could be a phase modulation of the motor region by the premotor region, in which the alpha phase in both regions is regulated by a corticothalamic loop and movement-specific information is passed from the inferior (the modulating) site to the superior (the modulated) site through the $80 \mathrm{~Hz}$ gamma phase, resulting in the $90 \mathrm{~Hz}$ phase in the modulated site. Given that we do not observe consistent single-band synchronization for the alpha rhythm, this seems unlikely. Alternatively, however, the frequencies in the modulating region could also originate from that region alone, and could simply be used to transmit taskrelated information in two independent "channels" to the modulated region. The interaction between the source and target site implies functional coupling between ventral premotor sources and primary sensorimotor target sites, which is well described in nonhuman primates (Geyer et al., 2000) and has been shown to play a role in human motor activity as well (de Graaf et al., 2008). The timing of this interaction just after movement onset leads us to speculate a role of these areas in monitoring movement, perhaps providing planning and feedback instructions with respect to an ongoing movement. The premotor site may correspond to the site of "mirror neurons" (Iacoboni and Dapretto, 2006) tuned to the observation of movement; thus, this observed interaction could represent the transfer of subject's interpretation of the visual observation of their own movement to sensorimotor regions. This would be particularly relevant to the planning of the end of the thumb movement or planning the subsequent repeti- 
tion. The need for on-line updating to the continuation or end of the visual cue to move the thumb also could be subserved by this mechanism. Future descriptions of phase modulation across other parts of the brain will help refine interpretation of this novel observation.

Given the vast space of possible channel and frequency combinations, our results, focusing only on the most significant interactions, should be seen and interpreted as an example of the complex interactions in the human sensorimotor system during voluntary finger movement. The existence of such interactions demonstrates that the human cortex is using a much wider range of communication between distant neuronal populations than can be revealed by single-band interactions. Consequently, multiplicative interaction and, more generally, phase-phase interactions may be a fundamental mode of communication between distant cortical areas.

\section{References}

Ahissar E, Kleinfeld D (2003) Closed-loop neuronal computations: focus on vibrissa somatosensation in rat. Cereb Cortex 13:53-62.

Buzsáki G, Draguhn A (2004) Neuronal oscillations in cortical networks. Science 304:1926-1929.

Canolty RT, Edwards E, Dalal SS, Soltani M, Nagarajan SS, Kirsch HE, Berger MS, Barbaro NM, Knight RT (2006) High gamma power is phaselocked to theta oscillations in humans neocortex. Science 313:1626-1628.

Chau W, McIntosh AR, Robinson SE, Schulz M, Pantev C (2004) Improving permutation test power for group analysis of spatially filtered MEG data. Neuroimage 23:983-996.

Crone NE, Miglioretti DL, Gordon B, Sieracki JM, Wilson MT, Uematsu S, Lesser RP (1998) Functional mapping of human sensorimotor cortex with electrocorticographic spectral analysis I. Alpha and beta event related desynchronization. Brain 121:2271-2299.

de Graaf JB, Frolov A, Fiocchi M, Nazarian B, Anton JL, Pailhous J, Bonnard M (2008) Preparing for a motor perturbation: early implication of primary motor and somatosensory cortices. Hum Brain Mapp. Advance online publication. Retrieved December 21, 2008. doi:10.1002/hbm.20526.

Efron B, Tibshirani R, Storey JD, Tusher V (2001) Empirical Bayes analysis of a microarray experiment. J Am Stat Assoc 96:1151-1160.

Fries P (2005) A mechanism for cognitive dynamics: neuronal communication through neuronal coherence. Trends Cogn Sci 9:474-480.

Gabbiani F, Krapp HG, Koch C, Laurent G (2002) Multiplicative computation in a visual neuron sensitive to looming. Nature 420:320-324.

Geyer S, Matelli M, Luppino G, Zilles K (2000) Functional neuroanatomy of the primate isocortical motor system. Anat Embryol 202:443-474.

Gross J, Timmermann L, Kujala J, Dirks M, Schmitz F, Salmelin R, Schnitzler A (2002) The neural basis of intermittent motor control in humans. Proc Natl Acad Sci U S A 99:2299-2302.
Iacoboni M, Dapretto M (2006) The mirror neuron system and the consequences of its dysfunction. Nat Rev Neurosci 7:942-951.

Kahana MJ (2006) The cognitive correlates of human brain oscillations. J Neurosci 26:1669-1672.

Lachaux JP, Rodriguez E, Martinerie J, Varela FJ (1999) Measuring phase synchrony in brain signals. Hum Brain Mapp 8:194-208.

Miller KJ, Leuthardt EC, Schalk G, Rao RP, Anderson NR, Moran DW, Miller JW, Ojemann JG (2007a) Spectral changes in cortical surface potentials during motor movement. J Neurosci 27:2424-2432.

Miller KJ, denNijs M, Shenoy P, Miller JW, Rao RP, Ojemann JG (2007b) Real-time functional brainmapping using electrocorticography. Neuroimage 37:504-507.

Mormann F, Fell J, Axmacher N, Weber B, Lehnertz K, Elger CE, Fernández G (2005) Phase/amplitude reset and theta-gamma interaction in the human medial temporal lobe during a continuous word recognition memory task. Hippocampus 15:890-900.

Nikias CL, Mendel JM (1993) Signal processing with higher-order spectra. IEEE Signal Process Mag 10:10-37.

Ohara S, Mima T, Baba K, Ikeda A, Kunieda T, Matsumoto R, Yamamoto J, Matsuhashi M, Nagamine T, Hirasawa K, Hori T, Mihara T, Hashimoto N, Salenius S, Shibasaki H (2001) Increased synchronization of cortical oscillatory activities between human supplementary motor and primary sensorimotor areas during volunatry movements. J Neurosci 21:9377-9386.

Palva JM, Palva S, Kaila K (2005) Phase synchrony among neuronal oscillations in the human cortex. J Neurosci 25:3962-3972.

Palva S, Palva JM (2007) New vistas for alpha-frequency band oscillations. Trends Neurosci 30:150-158.

Pantazis D, Nichols TE, Baillet S, Leahy RM (2005) A comparison of random field theory and permutation methods for the statistical analysis of MEG data. Neuroimage 25:383-394.

Rudrauf D, Douiri A, Kovach C, Lachaux JP, Cosmelli D, Chavez M, Adam C, Renault B, Martinerie J, Le Van Quyen M (2006) Frequency flows and the time-frequency dynamics of multivariate phase synchronizations in brain signals. Neuroimage 31:209-227.

Schack B, Vath N, Petsche H, Geissler HG, Möller E (2002) Phase-coupling of theta-gamma EEG rythms during short-term memory processing. Int J Psychophysiol 44:143-163.

Shils JL, Litt M, Skolnick BE, Stecker MM （1996）Bispectral analysis of visual interactions in humans 98:113-125.

Simoes C, Jensen O, Parkkonen L, Hari R (2003) Phase locking between human primary and secondary somatosensory cortices. Proc Natl Acad Sci U S A 100:2691-2695.

Tass P, Rosenblum MG, Weule J, Kurths J, Pikovsky A, Volkmann J, Schnitzler A, Freund HJ (1998) Detection of n:m phase locking from noisy data: application to magnetoencephalography. Phys Rev Lett 81:3291-3294

Zacksenhouse M, Ahissar E (2006) Temporal decoding by phase-locked loops: unique features of circuit-level implementations and their significance for vibrissal information processing. Neural Comput 18:1611-1636. 